\title{
The predictive value of procalcitonin, C-reactive protein, presepsin, and soluble-triggering receptor expressed on myeloid cell levels in bloodstream infections in pediatric patients with febrile neutropenia
}

\author{
Zeynep Canan Özdemirr1, Yeter Düzenli-Kar¹, Ağgül Canik², Zeynep Küskü-Kiraz², \\ Hülya Özen ${ }^{3}$, Özcan Bör ${ }^{1}$ \\ ${ }^{1}$ Division of Pediatric Hematology Oncology, Departments of ${ }^{2}$ Clinical Biochemistry and ${ }^{3}$ Bioistatistics, Eskişehir \\ Osmangazi University Faculty of Medicine,26480, Eskişehir, Turkey. E-mail: efecanan@yahoo.com
}

Received: 21st March 2018, Revised: 28th July 2018, Accepted: 31 st October 2018

SUMMARY: Özdemir ZC, Düzenli-Kar Y, Canik A, Küskü-Kiraz Z, Özen H, Bör Ö. The predictive value of procalcitonin, C-reactive protein, presepsin, and soluble-triggering receptor expressed on myeloid cell levels in bloodstream infections in pediatric patients with febrile neutropenia. Turk J Pediatr 2019; 61: 359-367.

The present study investigates the predictive value of procalcitonin (PCT), C-reactive protein (CRP), presepsin (PRE-SEP) and soluble-triggering receptor, as expressed on myeloid cells (sTREM-1) levels in bloodstream infections in pediatric patients with febrile neutropenia. A total of 47 episodes of febrile neutropenia that developed in 30 children with malignancy were analyzed in this study, while the control group comprised 27 children who had undergone chemotherapy for malignancy (completed $\geq 2$ years ago) without neutropenia, fever or drug use. Median PCT, CRP, PRE-SEP and sTREM-1 levels on admission were found to be significantly higher in the patient group than in the control group, while in the blood cultures, the microbiological agent was isolated in $13(27.7 \%)$ of the 47 episodes. Median PCT and CRP levels on days 1, 2 and 7 were higher in the blood culture-positive episodes than in the blood culture-negative episodes. There was no significant difference in the PRE-SEP and STREM-1 levels on days 1, 2 and 7 between the blood culture-positive and blood culture-negative episodes. The results of the study suggest that PRESEP and STREM-1 are at measurable levels upon admission in children with febrile neutropenia, but that these markers may not be appropriate for the predicting of bloodstream infections, although CRP and PCT levels within the first 24 hours may serve as a guide for clinicians.

Key words: febrile neutropenia, procalcitonin, C-reactive protein, presepsin, solubletriggering receptor expressed on myeloid cells.

Infectious complications in pediatric oncology patients are a major cause of morbidity and mortality. ${ }^{1}$ There have been many studies investigating the potential use of various biomarkers to differentiate between patients at high-risk of bacteremia or severe bacterial infection during febrile neutropenia episodes. ${ }^{2}$

CD14 is a receptor for the lipopolysaccharidelipopolysaccharide binding protein that can be found on the surfaces of monocytes, macrophages and, in lesser amounts neutrophils. Presepsin (PRE-SEP) is soluble form of CD14..$^{3-5}$ When a pro-inflammatory cascade is activated against infectious agents, it is released following phagocytosis or released into circulation by breakdown of CD14 via the proteolytic pathway. ${ }^{4,5}$ Its high specificity for bacterial infections is one of its most important characteristics. ${ }^{6}$ Several studies have shown that PRE-SEP is superior to procalcitonin (PCT) and C-reactive protein 
(CRP) in diagnosing sepsis. ${ }^{4}$ Its role in febrile neutropenic patients, however, has not been well-established and there is little data on febrile neutropenic children.

The triggering receptor expressed on myeloid cells (TREM-1) plays a role in the regulation of natural immunity and signals induced by Toll-like receptors. ${ }^{7}$ An increased expression of soluble-triggering receptor is expressed on myeloid cell (sTREM-1) in bacterial and fungal infections, ${ }^{8}$ and in addition, sepsis induces sTREM-1, which can be detected in biological fluids. ${ }^{7}$ sTREM-1 has been designated as a potentially effective marker in bacterial infections, ${ }^{9}$ although there is, however, only limited data on the role of sTREM-1 in the diagnosis and follow-up of sepsis in pediatric patients.

Although several studies have reported the superiority of PCT over CRP in predicting bacterial infections and a poor clinical course in pediatric patients, the data reported in the literature is conflicting. ${ }^{10-12}$ For many years, CRP has been used as a marker of sepsis in pediatric patients, however it has only limited specificity. ${ }^{2}$ The accuracy of CRP alone in predicting severe bacterial infections (i.e., sepsis, severe sepsis) in febrile neutropenic children has been found to be lower than other markers, such as interleukin (IL) -6 and PCT. ${ }^{13}$ Despite extensive efforts, there is no widely accepted means of identifying patients at high-risk of bacterial infections in febrile neutropenia.

In the present study, we investigate the predictive value of PCT, CRP, PRE-SEP and sTREM-1 levels in bloodstream infections in pediatric patients with febrile neutropenia.

\section{Material and Methods}

A total of 44 children who underwent chemotherapy due to hematological or solid organ malignancy between December 2015 and February 2016 were included in the study.
Febrile neutropenia diagnosed in the event of axillary temperature measuring $>38^{\circ} \mathrm{C}$ on one occasion or $>37.5^{\circ} \mathrm{C}$ for at least one hour in patients with an absolute neutrophil count (ANC) of $<0.5 \times 10^{9} / \mathrm{L}$, or who had an ANC of between 0.5 and $1 \times 10^{9} / \mathrm{L}$, and whose ANC was expected to decrease below $0.5 \times 10^{9} / \mathrm{L}$ within 24-48 hours. ${ }^{14}$ Data on the age and sex of the participants, vital signs, duration of fever, blood count parameters, physical examination findings and culture results was obtained from medical charts. Febrile neutropenia episodes were classified into two groups, depending on the isolation of the microbiological agent from the blood culture. Irrespective of the infectious foci, episodes in which a microbiologic agent was isolated from the blood culture were defined as culture-positive episodes (i.e., bacteria or fungi), while episodes in which no microbiologic agent was isolated were defined as culture-negative episodes. The presence of sepsis was clinically defined as the cardiac apex beat and respiratory rate being $>2$ standard deviation (SD) according to age. ${ }^{15}$ The control group was composed of 27 children who were in remission and who had completed chemotherapy at least two years prior to the study, and who had no neutropenia, infection or drug use. The study was approved by the ethics committee (ethic approval no: 80558721/04), and was conducted in accordance with the principles of Helsinki. Written informed consent was obtained from the legal guardians of the participant children.

\section{Exclusion criteria}

Those who showed no signs of disease remission in the bone marrow $(n=2)$ and those who received a granulocyte colony-stimulating factor (G-CSF) or antibiotics at the onset of febrile neutropenia episodes $(n=5)$ were excluded from the study. Furthermore, two patients refused to participate, and laboratory analyses were missing for five more, and so they were excluded from the final analysis. A flow diagram is presented in Figure 1. 


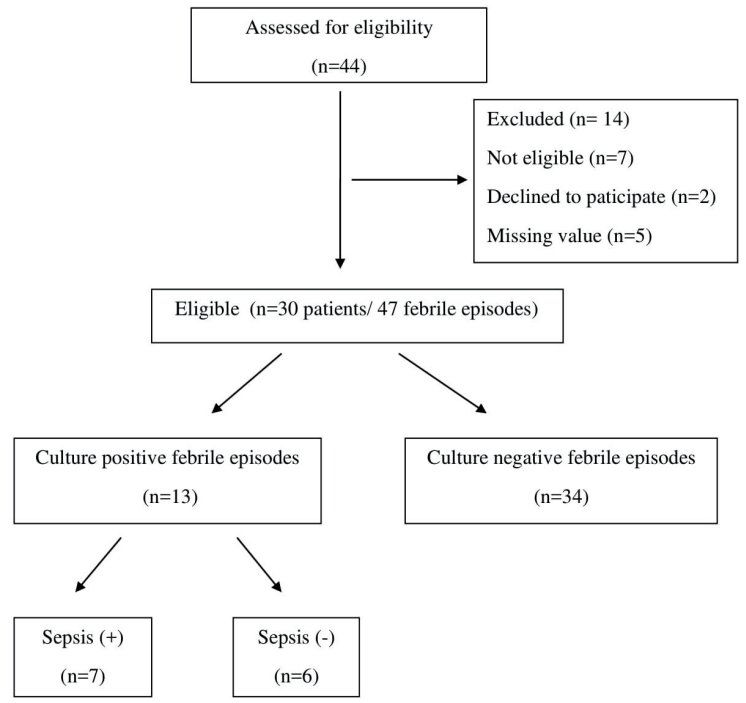

Fig. 1. Recruitment diagram for study.

\section{Sampling and laboratory analysis}

Each patient provided three blood samples, the first within 24 hours of admission (day 1 ), the second between 24 and 48 hours (day 2 ), and the third on day 7. Only one blood sample was obtained from the control group. The blood samples were collected from a peripheral vein into vacutainers containing ethylenediaminetetraacetic acid (EDTA) and gel tubes. The complete blood count was analyzed using an automated cell counter (Beckman Coulter Gen-S SM, USA), while PCT concentrations were measured using an electrochemiluminescence immunoassay (ECLIA) using a Cobas e411 device (Roche Diagnostics, Mannheim, Germany). The lower detection limit of this method was $\leq 0.02 \mathrm{ng} /$ $\mathrm{mL}$ and the inter-assay coefficient of variability (CV) was 2.1 percent. CRP levels were measured using the nephelometric method using a Siemens BNII (Healthcare, Munich, Germany) auto-analyzer.

For the PRE-SEP and sTREM-1 measurements, serum samples were separated from the cells by centrifugation at 2,000xg for $10 \mathrm{~min}$, and the samples were stored at $-80^{\circ} \mathrm{C}$ until analysis. Serum PRE-SEP and STREM-1 levels were measured using enzyme-linked immunosorbent assays (ELISA) using a VICTOR X3 Perkinelmer ELISA device (Perkin Elmer,Waltham, MA, USA). SunRed (Shanghai, China) brand kits were used for PRE-SEP measurement, and a Cusabio (Wuhan, China) brand kit was used for sTREM-1 measurement. The lower detection limit for the measurement of PRE-SEP was $0.03 \mathrm{mg} / \mathrm{L}$, the intra-assay $\mathrm{CV}$ was $<8$ percent, and the inter-assay $\mathrm{CV}$ was $<10$ percent. The lower detection limit for the measurement of sTREM-1 was $31.25 \mathrm{pg} / \mathrm{mL}$ with $<8$ percent intra-assay $\mathrm{CV}$ and a $<10$ percent inter-assay CV.

\section{Statistical analysis}

The statistical analysis was carried out using SPSS version 21.0 statistical software (IBM Corp., Armonk, NY, USA). Descriptive statistics were expressed as mean $\pm S D$ for normally distributed variables, and in the median and interquartile range for other variables. Categorical data was compared using a Chi-square test, and a KolmogorovSmirnov test was used to analyze the normality of distribution for continuous variables. Normally distributed continuous variables were compared using an independent t-test, while a Mann-Whitney U-test was used to compare abnormally distributed variables. To identify the relationship between two variables, a Spearman's correlation coefficient was calculated, and to evaluate whether serum PCT, CRP, PRE-SEP and sTREM-1 levels could be used to accurately diagnose bloodstream infections, receiver operating characteristic (ROC) curves were used. Differences were considered statistically significant when $\mathrm{P}$ values $<0.05$.

\section{Results}

A total of 47 febrile neutropenic episodes of 30 patients were included in the present study. Seventeen patients $(56.6 \%)$ had acute lymphoblastic leukemia, five $(16.6 \%)$ had non-Hodgkin lymphoma, two $6.7 \%$ ) had acute myeloid leukemia, two $(6.7 \%)$ had Hodgkin's lymphoma, two $(6.7 \%)$ had a Burkitt leukemia, and two $(6.7 \%)$ had Wilms tumors. A bacterial or fungal agent was identified from positive blood cultures in 13 of the 47 (27.7\%) febrile neutropenic episodes, Methicillin-resistant coagulase-negative Staphylococcus aureus was isolated in two of the 13 cultures, coagulasenegative Staphylococcus aureus in three 
Table I. Clinical Characteristics and Laboratory Parameters of the Patients and the Control Group.

\begin{tabular}{lccc}
\hline & $\begin{array}{c}\text { Patient group } \\
(\mathrm{n}=30)\end{array}$ & $\begin{array}{c}\text { Control group } \\
(\mathrm{n}=27)\end{array}$ & $\mathrm{p}$ \\
\hline Age $($ years $)$ & $8.6 \pm 0.83$ & $8.30 \pm 0.56$ & $>0.05$ \\
Sex $($ Female/Male $)$ & $13 / 17$ & $15 / 12$ & $>0.05$ \\
Leukocyte count $\left(\times 10^{9} / \mathrm{L}\right)$ & $1.0(0.4-1.5)$ & $4.3(2.6-6.3)$ & $<0.001$ \\
Neutrophil count $\left(\times 10^{9} / \mathrm{L}\right)$ & $0.1(0-0.5)$ & $2.4(1.5-3.1)$ & $<0.001$ \\
PCT $(\mathrm{ng} / \mathrm{ml})$ & $0.29(0.13-0.80)$ & $0.07(0.04-0.08)$ & $<0.001$ \\
CRP $(\mathrm{mg} / \mathrm{dl})$ & $3.0(0.5-7.39)$ & $0.34(0.30-0.46)$ & $<0.001$ \\
PRE-SEP $(\mathrm{mg} / \mathrm{L})$ & $0.79(0.43-1.86)$ & $0.44(0.17-1.59)$ & 0.034 \\
sTREM-1 $(\mathrm{pg} / \mathrm{ml})$ & $36.59(6.74-8.16)$ & $4.18(2.21-18.09)$ & 0.001 \\
\hline
\end{tabular}

CRP: C-reactive protein, PRE-SEP:Presepsin, PCT:Procalcitonin, sTREM-1: Soluble-triggering receptor expressed on myeloid cell

cultures, Escherichia coli in two cultures, Klebsiella pneumonia in two cultures, Pseudomonas aeuriginosa in one culture, Acromobacter dentrificans in one culture, Geotrichum capitatum in one culture, Candida crusei in one culture and Candida parapsilosis in one culture. Of the total, $34(72.3 \%)$ of the febrile neutropenia episodes were culturenegative.

Of the patients, 17 were boys and 13 were girls, with a mean age of $8.60 \pm 0.83$ years. Of the patients in the control group, 12 were boys and 15 were girls, with a mean age of $8.30 \pm 0.56$ years. The distribution of age and sex was similar between the patient and control groups ( $p>0.05$ for both). The median leucoyte and neutrophil count were significantly lower than in the control group ( $\mathrm{p}<0.001$ for both). The median PCT, CRP, PRE-SEP and sTREM-1 levels of the study patients were at the onset of febrile neutropenia episodes were significantly higher than the values in the control group $(\mathrm{p}<0.001, \mathrm{p}<0.001, \mathrm{p}<0.05, \mathrm{p}=0.001$, respectively) (Table I).

CRP values in the culture-positive episodes on days 1, 2 and 7 were significantly higher than culture-negative episodes $(\mathrm{p}<0.01, \mathrm{p}=0.001$, $\mathrm{p}<0.05$, respectively). In addition, PCT values in culture-positive episodes on days 1,2 and 7 were significantly higher than culturenegative episodes $(\mathrm{p}<0.05$ for each). There was no significant difference between the culture-positive and culture-negative episodes in terms of PRE-SEP and sTREM-1 levels on days 1,2 and 7 ( $p>0.05$, for each). The median duration of fever was significantly longer in the culture-positive episodes when compared to the culture negative episodes $(\mathrm{p}<0.05)$ (Table II).

There were clinical findings of sepsis in seven of the 13 culture-positive episodes. The median CRP and PCT values on days 2 and 7 in episodes showing signs of sepsis were significantly higher than the values in episodes showing no signs of sepsis $(\mathrm{p}<0.05$ for each; and $\mathrm{p}<0.05, \mathrm{p}<0.01$ respectively). The median sTREM-1 levels of patients showing signs of sepsis on days 1, 2 and 7 were higher than the values in patients showing no signs of sepsis, although the difference was not statistically significant ( $p>0.05$, for each). There was also no difference between the two groups with respect to PRE-SEP levels ( $p>0.05$, for each) (Table III).

The ROC was used to determine cut-off values for CRP, PCT, PRE-SEP and STREM-1. The cut-off value for PCT was $0.5 \mathrm{ng} / \mathrm{mL}$ on days 1 and 7 , and $0.25 \mathrm{ng} / \mathrm{mL}$ on day 2 . The cut-off value for CRP was $2.5 \mathrm{mg} / \mathrm{dl}$ on day $1,4 \mathrm{mg} / \mathrm{dl}$ on day 2 , and $7 \mathrm{mg} / \mathrm{dl}$ on day 7 (Table IV). No sensitivity or specificity values were provided for PRE-SEP and sTREM-1, as they had a low power in the differentiation of culture-positive from culture negative episodes. The ROC curves for CRP and PCT are shown in Figure 2. 
Table II. Laboratory Parameters and Duration of Fever in Culture-Positive and Culture-Negative Episodes on Days 1, 2, and 7 .

\begin{tabular}{lccc}
\hline & $\begin{array}{c}\text { Culture-positive } \\
(\mathrm{n}=13)\end{array}$ & $\begin{array}{c}\text { Culture-negative } \\
(\mathrm{n}=34)\end{array}$ & $\mathrm{p}$ \\
\hline Leukocyte count (Day 1) & $0.6(0.25-1.15)$ & $1.15(0.6-1.52)$ & $<0.05$ \\
Leukocyte count (Day 2) & $0.5(0.3-1.0)$ & $1.1(0.57-1.72)$ & $<0.05$ \\
Leukocyte count (Day 7) & $1.3(0.5-6.75)$ & $2.3(1.35-4.5)$ & $>0.05$ \\
\hline Neutrophil count (Day 1) & $0.1(0-0.2)$ & $200(0-625)$ & $>0.05$ \\
Neutrophil count (Day 2) & $0.1(0-0.15)$ & $0.15(0-0.65)$ & $>0.05$ \\
Neutrophil count (Day 7) & $1.0(0.1-4.35)$ & $0.9(0.30-1.92)$ & $>0.05$ \\
\hline CRP (Day 1) & $6.79(2.87-17.0)$ & $1.91(0.36-5.51)$ & $<0.01$ \\
CRP (Day 2) & $12.0(5.31-16.50)$ & $2.94(0.84-7.51)$ & 0.001 \\
CRP (Day 7) & $11.30(0.76-32.80)$ & $0.79(0.34-3.48)$ & 0.011 \\
\hline Procalcitonin (Day 1) & $0.60(0.25-2.24)$ & $0.21(0.11-0.45)$ & 0.021 \\
Procalcitonin (Day 2) & $0.62(0.30-2.90)$ & $0.20(0.13-0.89)$ & 0.044 \\
Procalcitonin (Day 7) & $0.57(0.14-15.0)$ & $0.18(0.09-0.41)$ & 0.034 \\
\hline Presepsin (Day 1) & $0.67(0.39-1.72)$ & $0.85(0.44-2.18)$ & $>0.05$ \\
Presepsin (Day 2) & $0.98(0.39-5.06)$ & $0.76(0.55-2.27)$ & $>0.05$ \\
Presepsin (Day 7) & $1.67(0.91-3.70)$ & $0.93(0.33-2.18)$ & $>0.05$ \\
\hline sTREM-1 (Day 1) & $50.89(8.65-81.56$ & $33.43(2.38-69.23)$ & $>0.05$ \\
sTREM-1 (Day 2) & $47.57(6.19-140.26)$ & $30.29(3.83-86.04)$ & $>0.05$ \\
sTREM-1 (Day 7) & $14.84(3.93-98.80)$ & $17.68(2.56-105.41)$ & $>0.05$ \\
\hline Duration of fever (hour) & $96(32-240)$ & $48(14.75-72)$ & $<0.05$ \\
\hline CRP- C-reave $)$ & \\
\hline
\end{tabular}

CRP: C-reactive protein, PRE-SEP:Presepsin, sTREM-1: Soluble-triggering receptor expressed on myeloid cell

Table III. Laboratory Parameters in Episodes With or Without Signs of Sepsis.

\begin{tabular}{lccc}
\hline & $\begin{array}{c}\text { Sepsis }(+) \\
(\mathrm{n}=7)\end{array}$ & $\begin{array}{c}\text { Sepsis }(-) \\
(\mathrm{n}=6)\end{array}$ & $\mathrm{p}$ \\
\hline CRP (Day 1) & $19.5(6.86-27.75)$ & $3.96(0.6-9.04)$ & $>0.05$ \\
CRP (Day 2) & $15.45(7.61-17.0)$ & $6.63(3.57-11.70)$ & 0.041 \\
CRP (Day 7) & $17.5(5.01-28.95)$ & $1.67(0.45-9.25)$ & 0.015 \\
\hline PCT (Day 1) & $0.80(0.5-1.6)$ & $0.25(0.09-1.8)$ & $>0.05$ \\
PCT (Day 2) & $0.66(0.60-5.72)$ & $0.30(0.12-1.03)$ & 0.022 \\
PCT (Day 7) & $15.0(2.39-80.25)$ & $0.15(0.08-0.35)$ & $<0.01$ \\
\hline PRE-SEP (Day 1) & $0.32(0.25-0.52)$ & $1.11(0.52-2.14)$ & $>0.05$ \\
PRE-SEP (Day 2) & $0.70(0.32-3.78)$ & $1.14(0.25-6.35)$ & $>0.05$ \\
PRE-SEP (Day 7) & $2.08(0.84-4.39)$ & $1.37(0.35-3.03)$ & $>0.05$ \\
\hline sTREM-1 (Day 1) & $165.15(22.77-429.90)$ & $5.26(1.30-15,63)$ & $>0.05$ \\
sTREM-1 (Day 2) & $136.27(26.19-210.86)$ & $29.76(1.30-59.60)$ & $>0.05$ \\
sTREM-1 (Day 7) & $104.84(6.57-420.09)$ & $15.80(2.83-79.20)$ & $>0.05$ \\
\hline
\end{tabular}

CRP: C-reactive protein, PRE-SEP:Presepsin, sTREM-1: Soluble-triggering receptor expressed on myeloid cell 
Table IV. Sensitivity and Specificity for CRP and PCT on Days 1,2 and 7 to Distinguish Culture Positive Episodes From Culture Negative Episodes.

\begin{tabular}{lcccccccc}
\hline \multicolumn{9}{c}{ CRP } \\
\hline & Cut-off & AUC(\%95CI) & Sensitivity & Spesificity & Cut-off & AUC(\%95CI) & Sensitivity & Spesificity \\
\hline Day 1 & 2.5 & 0.758 & 84.6 & 55.9 & 0.5 & 0.722 & 61.54 & 89.41 \\
Day 2 & 4 & 0.807 & 92.31 & 47.1 & 0.25 & 0.691 & 84.62 & 55.88 \\
Day 7 & 7 & 0.742 & 61.54 & 85.29 & 0.5 & 0.700 & 53.85 & 85.29 \\
\hline
\end{tabular}

CRP: C-reactive protein, PCT: Procalcitonin

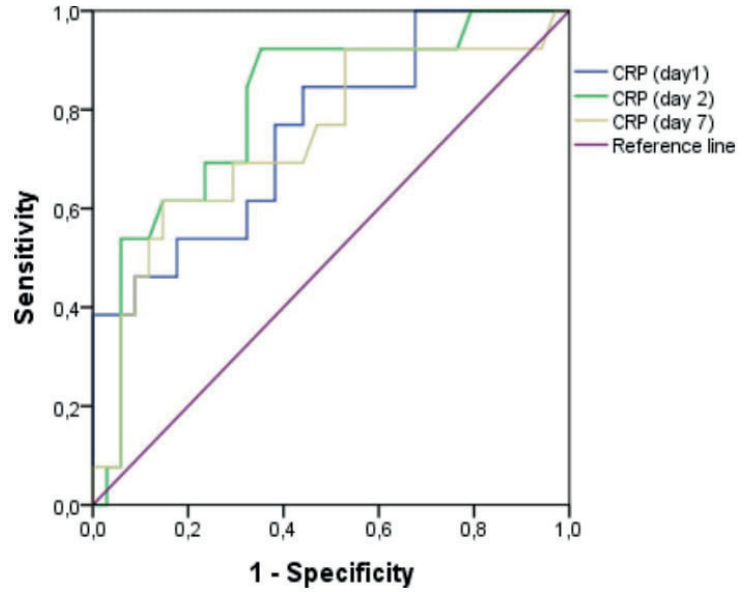

Fig. 2A. Receiver operating characteristic (ROC) curves of serum levels of CRP.

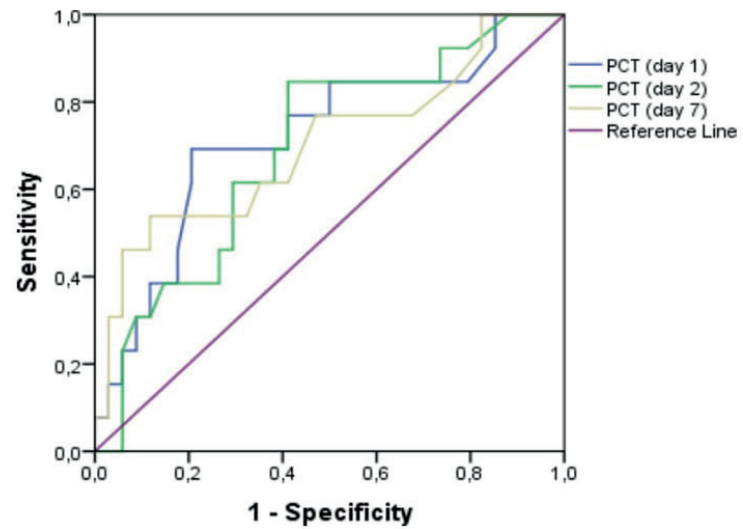

Fig. 2B. Receiver operating characteristic (ROC) curves of serum levels of PCT.

A negative correlation was noted between the neutrophil count and CRP and PRE-SEP values on day 1 ( $\mathrm{r}:-0.398, \mathrm{p}=0.006$ and $\mathrm{r}$ :.389, $\mathrm{p}=0.008)$, a positive correlation was noted between neutrophil count and STREM-1 $(\mathrm{r}: 0.418, \mathrm{p}=0.004)$, a positive correlation was noted between CRP and PCT $(r:+0.524$, $\mathrm{p}<0.001$ ), and a negative correlation was noted between PRE-SEP and STREM-1 ( $\mathrm{r}$ :$0.345, p=0.020)$.
A negative correlation was noted between neutrophil count and CRP on day 2 (r:-0.404, $\mathrm{p}=0.006)$, a positive correlation was noted between CRP and PCT ( $\mathrm{r}:+0.559, \mathrm{p}<0.001)$, and a positive correlation was noted between PCT and sTREM-1 (r:+0.348, p=0.019). A positive correlation was noted between CRP and PCT on day $7(\mathrm{r}:+0.497, \mathrm{p}=0.001)$ and a positive correlation was noted between CRP and PRE-SEP ( $\mathrm{r}:+0.492, \mathrm{p}=0.001)$.

\section{Discussion}

Early diagnosis and the appropriate treatment of infections significantly affects the prognosis of patients with febrile neutropenia. Culture analyses are considered to be the optimum method in the diagnosis of infections, ${ }^{11}$ although the likelihood of demonstrating a causative agent is low, and it takes long time to obtain culture results. ${ }^{1}$ Diagnostic tests are required for the swift diagnosis of fever of infectious origin, and there are ongoing studies investigating the use of various markers. In the present study, we found that CRP and PCT were useful markers in predicting bloodstream infections in pediatric patients with febrile neutropenia, although PRE-SEP and STREM-1 proved to be of low significance.

Literature contains a limited number of studies on this subject, as there are concerns that PRE-SEP levels cannot be measured or may give false-negative results in patients with hematological malignancies, since high doses of chemotherapy induce severe cytopenia, including CD14-expressing neutrophils and monocytes. Previous studies have yielded various results. Ebihara et al. ${ }^{16}$ found that PRESEP could be of low diagnostic significance in neutropenic patients, and Koizumi et al. ${ }^{6}$ showed that the cut-off levels for PRE-SEP 
in gram-negative bacteremia were higher than the cut-off levels at the onset of febrile neutropenia. Maurice et al. ${ }^{17}$ identified a positive correlation between the severity of sepsis and PRE-SEP levels, and that PRE-SEP was more specific than CRP in identifying sepsis, while Koh et al. ${ }^{18}$ reported that PRESEP was a more sensitive marker of bacterial infection, than PCT, although the ability of PRE-SEP in differentiating septic shock from other conditions was lower than PCT.

Furthermore, in two studies in literature involving pediatric patients with febrile neutropenia, Urbonas et al. ${ }^{19}$ reported no significant difference in the PRE-SEP levels of pediatric patients with bacteremia and febrile neutropenia and patients with fever of unknown origin; while in a study by Olad et al., ${ }^{20}$ no difference was noted in the PRESEP levels of culture-positive and culturenegative episodes, while increased PRE-SEP levels were found to be correlated with the severity of infection. In the present study, we were able to measure the PRE-SEP levels in all children, despite severe neutropenia, and the PRE-SEP levels in the patient group were found to be significantly higher than in the control group. This finding may support the hypothesis that PRE-SEP is released primarily from monocytes rather than neutrophils, and that the production of PRE-SEP in tissue macrophages plays an important role in the maintenance of plasma PRE-SEP levels. ${ }^{6,21}$ On the other hand, the present study was unable to identify a relationship between PRE-SEP levels and severity of infection. Although the PRE-SEP levels slightly increased in repeated measurements in patients with the signs of sepsis, no statistically significant difference was identified between patients with and without signs of sepsis. The lack of difference in the PRE-SEP levels of culture-positive and culture-negative episodes may be related to severe neutropenia, decreased monocyte count, and also a decreased phagocytosis of the pathogenic agents and, thus, suppression of PRE-SEP production.

There is a lack of consensus on the value of sTREM-1 in predicting bacterial infections. ${ }^{22,23}$ There is an increasing body of evidence showing increased sTREM levels in biological fluids in the absence of an infection, ${ }^{24}$ while in a review study by Baraud et al.,24 increased levels were reported in non-infectious inflammatory diseases, such as acute pancreatitis, inflammatory bowel disease, vasculitis and auto-immune diseases. Studies including adult patients tended to suggest a possible relationship between clinical course and sTREM-1 levels. Gibot et al. ${ }^{22}$ reported lower sTREM-1 levels upon admission in adult non-survivors from sepsis when compared to survivors, whereas the sTREM-1 levels remained stable or increased in patients who survived for two weeks. In contrast, Jeong et al. ${ }^{25}$ found higher sTREM-1 levels in nonsurvivors both upon admission and during follow-up, and Kwofie et al. ${ }^{26}$ also showed increased sTREM-1 levels in parallel to the increasing severity of the clinical condition, despite severe neutropenia in adults with febrile neutropenia. Furthermore, two studies including pediatric patients with febrile neutropenia yielded controversial results: Miedema et al..$^{27}$ suggested that sTREM-1 levels were below the detectable limit in the majority of the patients, and concluded that STREM-1 was not a useful marker for predicting bacterial infections; while Arzanian et al. ${ }^{28}$ reported that sTREM-1 showed a high sensitivity $(85 \%)$ and specificity $(98 \%)$ in predicting bloodstream infections. In the present study, we were able to measure PRE-SEP and sTREM-1 levels, despite severe neutropenia, and the sTREM-1 levels in the patients were found to be higher than in the control group. However, there was no significant difference in the sTREM-1 levels of the patients with (culture-positive) and without (culturenegative) bloodstream infections on days 1, 2 and 7 . In addition, the ROC curve revealed a low power in differentiating between patients with infections. This finding leads us to suggest that STREM-1 levels have little value in predicting bloodstream infections. On the other hand, despite the lack of statistical significance, the remarkably higher median sTREM-1 levels in patients with clinical signs of sepsis suggest that the sTREM-1 may be a potential marker for the prediction of the clinical course. These results, however, should be further investigated in more comprehensive studies. 
PCT has been suggested as a marker with a higher specificity than CRP in differentiating between fever caused by infections from those of unknown origin in patients with febrile neutropenia. ${ }^{29}$ Urbonas et al. ${ }^{19}$ demonstrated the diagnostic value of PCT in bacteremia or sepsis in pediatric patients with febrile neutropenia. However in a review of infants, children and adolescents conducted by Sakr et al., ${ }^{12}$ the authors failed to identify the actual role of PCT in febrile neutropenia. Lin et al., ${ }^{30}$ on the other hand, showed that CRP and PCT were comparable in terms of their diagnostic accuracy in detecting bacterial sepsis in children with febrile neutropenia. A meta-analysis reported that studies including pediatric patients were heterogeneous in terms of sample size and methodology, indicating the need for further studies investigating the utility of PCT. ${ }^{29}$ In the present study population, the PCT and CRP levels in patients with bloodstream infections were higher on days 1, 2 and 7 than those without bloodstream infections, while these sensitivity and specificity values within the first 24 hours were high. These results suggest that PCT and CRP may be useful markers in predicting infections, and one may not be superior to the other.

There are some limitations to this study that should be noted. First, the sample size was small, and febrile neutropenia episodes were not analyzed by classifying them according to the focus of infection. Second, although the present study aimed to evaluate whether better results could be obtained in predicting infections by combining CRP and PCT measurements with PRE-SEP and sTREM-1, no further evaluation was able to be made, as the PRE-SEP and STREM-1 showed a low power in differentiating between patients with infections.

In conclusion, despite severe neutropenia, both PRE-SEP and STREM-1 are secreted in sufficient amounts in children with febrile neutropenia, but PRE-SEP and STREM-1 lack sufficient diagnostic power to predict patients with bloodstream infections. On the other hand, CRP and PCT levels measured within the first 24 hours may guide treatment. Finally, although the present study failed to reach statistical significance, sTREM-1 levels can be considered a potential marker of clinical course.

\section{Acknowledgement}

This study was supported financially by The Commission for Scientific Research Projects (Project number 2016/732).

\section{REFERENCES}

1. Hann I, Viscoli C, Paesmans M, Gaya H, Glauser M. A comparison of outcome from febrile neutropenic episodes in children compared with adults: Results from four EORTC studies. International Antimicrobial Therapy Cooperative Group (IATCG) of the European Organization for Research and Treatment of Cancer (EORTC). Br J Haematol 1997; 99: 580-588.

2. Lanziotti VS, Povoa P, Soares M, Silva JR, Barbosa AP, Salluh JIF. Use of biomarkers in pediatric sepsis: Literature review. Rev Bras Ter Intensiva 2016; 28 : 472-482.

3. Grunwald U, Krüger C, Westermann J, Lukowsky A, Ehlers M, Schütt C. An enzyme-linked immunosorbent assay for the quantification of solubilized CD14 in biological fluids. J Immunol Methods 1992; 155: 225 232.

4. Yaegashi Y, Shirakawa K, Sato N, et al. Evaluation of a newly identified soluble CD14 subtype as a marker for sepsis. J Infect Chemother 2005; 11: 234-238.

5. Ackland GL, Prowle JR. Presepsin: Solving a soluble (CD14) problem in sepsis? Intensive Care Med 2015; 41: 351-353.

6. Koizumi Y, Shimizu K, Shigeta M, et al. Plasma presepsin level is an early diagnostic marker of severe febrile neutropenia in hematologic malignancy patients. BMC Infect Dis 2017; 17: 27.

7. Ford JW, McVicar DW. TREM and TREM-like receptors in inflammation and disease. Curr Opin Immunol 2009; 21: 38-46.

8. Bleharski JR, Kiessler V, Buonsanti C, et al. A role for triggering receptor expressed on myeloid cells-1 in host defense during the early-induced and adaptive phases of the immune response. J Immunol 2003; 170: 3812-3818.

9. Jiyong J, Tiancha H, Wei C, Huahao S. Diagnostic value of the soluble triggering receptor expressed on myeloid cells-1 in bacterial infection: A metaanalysis. Intensive Care Med 2009; 35: 587-595.

10. Fioretto JR, Martin JG, Kurokawa CS, et al Comparison between procalcitonin and C-reactive protein for early diagnosis of children with sepsis and septic shock. Inflamm Res 2010; 59: 581-586. 
11. Simon, L, Gauvin F, Amre DK, Saint-Louis P, Lacroix J. Serum procalcitonin and C-reactive protein levels as markers of bacterial infection: A systematic review and meta-analysis. Clin Infect Dis 2004; 15: 206-217.

12. Sakr Y, Sponholz C, Tuche F, Brunkhorst F, Reinhart $\mathrm{K}$. The role of procalcitoninin febrile neutropenic patients:Review of the literature. Infection 2008; 36: 396-407.

13. Kitanovski L, Jazbec J, Hojker S, Derganc M. Diagnostic accuracy of lipopolysaccharide-binding protein for predicting bacteremia/ clinical sepsis in children with febrile neutropenia: Comparison with interleukin-6, procalcitonin, and C-reactive protein. Support Care Cancer 2014; 22: 269-277.

14. Kebudi R, Devecioğlu Ö, Gürler N. Pediatrik febril nöropeni kılavuzu: Tanımlar ve tanı yöntemleri. Flora 2004; 2: 73-105.

15. Randolph AG, McCulloh RJ. Pediatric sepsis: Important considerations for diagnosing and managing severe infections in infants, children, and adolescents. Virulence 2014; 5: 179-189.

16. Ebihara Y, Kobayashi K, Ishida A, et al. Diagnositc performance of procalcitonin, presepsin and Creactive protein in patients with hematological malignancies. J Clin Lab Anal 2017; 31: e22147.

17. Maurice M, Nafea D, Sawy M, Soelam R, Youssef S. Usefulness of Presepsin (Soluble CD14 Subtype) as a diagnostic marker of sepsis in Egyptian patients with acute myeloid leukemia. Am J Mol Biol 2014; 4: 169176.

18. Koh H, Aimoto $M$, Katayama $T$, et al. Diagnostic value of levels of presepsin (soluble CD-14 subtype) in febrile neutropenia in patients with hematological disorders. J Infect Chemother 2016; 22: 466-471.

19. Urbonas V, EidukaiteA, Tamuliene I. The predictive value of soluble biomarkers (CD14 subtype, interleukin-2 receptor, human leukocyte antigen-G) and procalcitonin in the detection of bacteremia and sepsis in pediatric oncology patients with chemotherapy-induced febrile neutropenia. Cytokine 2013; 62: 34-37.

20. Olad E, Sedighi I, Mehrvar A, et al. Presepsin (Scd14) as a marker of serious bacterial infections in chemotherapy induced severe neutropenia. Iran J Ped 2014; 24: 715-722.

21. Arai Y, Mizugishi K, Nonomura K, Naitoh K, TakaoriKondo A, Yamashita K. Phagocytosis by human monocytes is required for the secretion of presepsin. J Infect Chemother 2015; 21: 564-569.
22. Gibot S, Le Renard PE, Bollaert PE, et al. Surface triggering receptor expressed on myeloid cells 1 expression patterns in septic shock. Intensive Care Med 2005; 31: 594-597.

23. Rivera-Chavez FA, Minei JP. Soluble triggering receptor expressed on myeloid cells-1 is an early marker of infection in the surgical intensive care unit. Surg Infect (Larchmt) 2009; 10: 435-439.

24. Barraud D, Gibot S. Triggering receptor expressed on myeloid cell 1. Crit Care Clin 2011; 27: 265-279.

25. Jeong SJ, Song YG, Kim CO, et al. Measurement of plasma sTREM-1 in patients with severe sepsis receiving early goal-directed therapy and evaluation of its usefulness. Shock 2012; 37: 574-578.

26. Kwofie L, Rapoport BL, Fickl H, et al. Evaluation of circulating soluble triggering receptor expressed on myeloid cells-1 (sTREM-1) to predict risk profile, response to antimicrobial therapy, and development of complications in patients with chemotherapyassociated febrile neutropenia: A pilot study. Ann Hematol 2012; 91: 605-611.

27. Miedema KG, de Bont ES, Elferink RF, et al. The diagnostic value of CRP, IL-8, PCT, and sTREM-1 in the detection of bacterial infections in pediatric oncology patients with febrile neutropenia. Support Care Cancer 2011; 19: 1593-1600.

28. Arzanian MT, Soltani B, Fahimizad A, Shiva F, Shamshiri AR, Karimi A. Association of serum soluble triggering receptor expressed on myeloid cells levels in malignant febrile neutropenic patients with bacteremia and fungemia. Iran J Pediatr 2011; 21: 301-306.

29. Phillips RS, Wade R, Lehrnbecher T, Stewart LA, Sutton AJ. Systematic review and meta-analysis of the value of initial biomarkers in predicting adverse outcome in febrile neutropenic episodes in children and young people with cancer. BMC Med 2012; 10: 6 .

30. Lin SG, Hou TY, Huang DH, et al. Role of procalcitoninin the diagnosis of severe infection in pediatric patients with fever and neutropenia-a systematic review and meta-analysis. Pediatr Infect Dis J 2012; 10: e182-e188. 